
FORMAÇÃO DE EDUCAÇÃO CIENTÍFICA

\title{
ASSESSMENT OF PRE-SERVICE PRIMARY TEACHERS' PEDAGOGICAL KNOWLEDGE IN ELEMENTARY SCIENCE: EFFECTS FROM SCIENCE EDUCATION TRAINING
}

\author{
VERDUGO-PERONA, José Javier ${ }^{1 *}$; SOLAZ-PORTOLÉS, Joan Josep ${ }^{2}$; SANJOSÉ, Vicente ${ }^{3}$ \\ ${ }^{1}$ Doctor por la Universitat de València (Spain).
}

${ }^{2,3}$ Universitat de València, Facultat de Magisteri, Departament de Didática de les Ciències Experimentals $\mathrm{i}$ Socials, Avinguda dels Tarongers 4, 46022 Valencia (Spain)

\author{
${ }^{*}$ Autor correspondente \\ e-mail: Joan.Solaz@uv.es
}

Received 23 July 2017; received in revised form 15 November 2017; accepted 15 November 2017

\section{RESUMO}

O Conhecimento Didático em ciências (CD) pode ser entendido como um conjunto de técnicas de análise educacional e de instrução, e de estratégias de administração que os professores usam para ensinar. Foi utilizado um instrumento validado para a sua avaliação. Este instrumento é usado para a análise do conhecimento pedagógico do conteúdo e é denominado ReCo. Além disso, foram definidos indicadores precisos (rubricas) combinando critérios fornecidos por especialistas e análise de dados de um estudo exploratório com professores em formação inicial. O grupo de indicadores mostrou suficiência para dar conta da variabilidade das respostas dos participantes. Estes indicadores foram utilizados para analisar os efeitos no CD derivados de instrução em didática geral versus didática das ciências. Os resultados mostram uma contribuição importante da formação específica em didática das ciências em CD de professores de primário em formação inicial.

Palavras-chave: Ensino das ciências; Formação de professores; Conhecimiento pedagógico em ciências; Avaliação; Indicadores.

\begin{abstract}
Pedagogical knowledge (PK) can be understood as the set of educational analysis and instructional technics; and also, management strategies that primary teachers use to teach. For its assessment, a previously validated instrument to assess Pedagogical Content Knowledge, called CoRe, was used and a set of specific indicators (rubrics) were defined combining experts' criteria and data analysis from an exploratory studio with preservice elementary teachers. Results showed the sufficiency of these rubrics to account for the variability of participants' answers. Those indicators were used to analyze effects on PK from the instruction in general versus specific didactic in science. Results show an important contribution from specific didactic in science on preservice elementary teachers' PK.
\end{abstract}

Keywords: Science education; Teachers education; Pedagogical knowledge in science; Assessment; Rubrics. 


\section{INTRODUCCIÓN}

Shulman (1987) estableció una serie de categorías de conocimiento básico que todo maestro/profesor y maestra/profesora debe poseer. De entre estas categorías destacan tres dominios que se mantienen a la vanguardia como conocimientos esenciales para una enseñanza efectiva de las ciencias: el Conocimiento del Contenido, el Conocimiento Didáctico y el Conocimiento Didáctico del Contenido (CDC) (ZEIDLER, 2002).

La enseñanza de una disciplina curricular requiere comprender y dominar su contenido (Conocimiento del Contenido), pero también saber transponer esos contenidos en prácticas instruccionales efectivas que incluyan técnicas de análisis educativo y estrategias de gestión. Es aquí donde se incluiría el Conocimiento Didáctico (CD) general y específico de los contenidos escolares, como es el caso de la ciencia. Este último podría entenderse como la parte instrumental del CDC que incluye también la parte disciplinar. De hecho, el CDC ha sido definido de un modo genérico como el conocimiento necesario para promover un aprendizaje efectivo de los contenidos, que suele ser fruto del tiempo, la formación y la experiencia del profesor (BARNETT y HODSON, 2001). El CDC caracteriza el conocimiento profesional propio de profesores (LOUGHRAN et al., 2006; Nilsson, 2008), y lo diferencia de otros profesionales con estudios disciplinares similares (por ejemplo, una física-profesora de un físicometeorólogo).

Se ha propuesto tomar el CDC como una guía para la formación inicial del profesorado, lo que favorecería la convergencia de metas formativas en Grados y Maestrías. La estructuración del currículo de formación inicial del profesorado tiene gran repercusión sobre las competencias adquiridas. Kleickmann et al. (2013) encontraron que la estructura del CDC adquirido por los futuros profesores reflejaba claramente la estructura de la formación universitaria proporcionada.

El objetivo básico de este trabajo es evaluar el conocimiento didáctico específico de ciencias escolares en futuros maestros de primaria. Sin embargo, realizar tal evaluación es una tarea difícil si no se atiende a algún contenido particular que permita enfocar la atención sobre aquello que se quiere analizar didácticamente y enseñar. En este caso serían temas de ciencia escolar, lo que nos acerca necesariamente al terreno de los estudios sobre Conocimiento Didáctico del Contenido (CDC), sobre el que se está trabajando profusamente a nivel internacional y del que podemos tomar planteamientos e instrumentos.

Se han diferenciado distintos componentes dentro del CDC (MAGNUSSON et al., 1999): orientaciones para la enseñanza, conocimiento del currículo, conocimiento de los estudiantes, conocimiento sobre evaluación, conocimiento sobre estrategias instruccionales. A partir de los componentes del CDC se han elaborado herramientas útiles para su evaluación. Algunos son cuestionarios de "lápiz y papel", de respuesta abierta (BRINES et al., 2016; KRAUSS et al., 2008; MANIZADE y MASON, 2011), o cerrada (JÜTTNER y NEUHAUS, 2013; JÜTTNER et al., 2013; MALCOLM y MAVHUNGA, 2015). Otros se basan en entrevistas y técnicas de observación usando indicadores de evaluación o "rúbricas" (PARK et al., 2008; GARDNER y GESSNEWSOME, 2011) O formularios semiestructurados (PARK y CHEN, 2012; HENZE et al., 2008), o registrando información mediante grabaciones y análisis de materiales usados por el profesor (ALONZO et al., 2012; FAIKHAMTA y CLARKE, 2013). También se han combinado ambos tipos de técnicas (ABD-EL-KHALICK, 2006; KÄPYLÄ et al., 2009; MORRISON y LUTTENEGGER, 2015).

De entre todos los instrumentos destacamos la herramienta ReCo o "Representación del Contenido" (LOUGHRAN et al., 2008; HUME y BERRY, 2011), que adaptaremos para nuestros propósitos. Para ello definiremos indicadores precisos que permitan detallar el contenido de las ideas de futuros maestros de primaria sobre didáctica en temas de ciencia escolar.

A pesar de la gran variedad de instrumentos, hasta donde llega nuestro conocimiento en el contexto español no se han realizado estudios de evaluación del Conocimiento Didáctico en ciencias con maestros en formación inicial usando indicadores precisos determinados a partir del análisis de las ideas de los propios protagonistas.

\section{OBJETIVOS}

Los objetivos específicos de este trabajo fueron:

O1: Adaptar el instrumento ReCo, ya validado en 
otros contextos, para evaluar el conocimiento básico en didáctica de las ciencias de futuros maestros. Definir indicadores ("rúbricas") para obtener una evaluación de los datos más precisa que la se obtiene a partir de las preguntas originales del instrumento, muy abiertas.

O2: Usar el instrumento adaptado para evaluar de forma exploratoria el conocimiento básico en didáctica de las ciencias de maestros de primaria en formación inicial.

O3: Valorar los efectos de una formación específica en didáctica de las ciencias experimentales sobre el conocimiento básico en didáctica de las ciencias en futuros maestros.

\section{MÉTODO}

\subsection{Participantes}

Participaron 112 alumnos/as de ambos sexos (22\% de hombres) pertenecientes a 4 grupos intactos del Grado de Maestro de Primaria en una universidad pública situada en una gran ciudad española. Su edad típica fue de 21 años (promedio de 21,1 y límites entre 20 y 42 años). Todos los participantes habían cursado y aprobado las materias troncales de formación psico-socio-pedagógica básica, y didáctica de carácter general. Además, también habían aprobado dos materias troncales sobre ciencias en esa universidad en particular: a) una dedicada a la formación en conocimiento científico que se cursa antes de la formación en didáctica de las ciencias (9 ects); b) otra dedicada a didáctica de las ciencias experimentales (9 ects).

Se procuró que la muestra de participantes incluyera diferentes niveles de conocimiento, interés y motivación hacia las temáticas de ciencias $(21 \%$ participantes tenía estudios previos al Grado especializados en ciencias experimentales).

Es importante tener en cuenta que el conocimiento básico en didáctica de las ciencias que puede ser expresado por estos/as participantes está aún desprovisto del importante componente de la maduración que proporciona la experiencia en el oficio.

\subsection{Instrumento}

Se seleccionó un instrumento procedente de la literatura especializada en Conocimiento Didáctico del Contenido, ya validado y utilizado en anteriores estudios: el ReCo (EAMES et al.,
2011). Este instrumento propone primero una situación o problema propio de ciencias y, a partir de ella, propone ocho grandes preguntas, todas ellas relacionadas con aspectos básicos de componentes del CDC, como por ejemplo: ¿Qué pretendes que los estudiantes aprendan sobre esta idea (la situación planteada)?; ¿Qué sabes sobre el pensamiento de los estudiantes que influye en la enseñanza de esta idea?

Para contestar esas grandes preguntas, el participante debe escribir libremente todo cuanto sepa, siempre respecto a la situación concreta propuesta al comienzo. En el presente estudio, se realizaron dos ajustes para adaptar el instrumento a los participantes:

a) Se clarificó la formulación de algunas preguntas con el uso de términos propios del oficio de maestro. Por ejemplo, la primera de las preguntas pasó a formularse así: ¿Qué objetivos concretos plantearías sobre esta situación para la educación de los/las niños/as?

b) Dos de las preguntas 0 aspectos del instrumento original, (3. ¿Qué otras cuestiones sobre la situación mencionada consideras relevantes?; y 6 . ¿Podrían influir otros factores a lo largo del proceso de enseñanza/aprendizaje de esta cuestión?) se subsumieron en otras preguntas por considerarse reiterativas. En una prueba piloto anterior solo dieron lugar a dudas de los estudiantes, y a respuestas repetidas, genéricas o inespecíficas.

El resultado fue un instrumento con seis aspectos diferenciados en los que se articula el conocimiento didáctico del contenido (siempre a excepción del componente "experiencia profesional"):

1) ¿Qué objetivos concretos plantearías sobre esta situación para la educación de los/las niños/as?

2) ¿Por qué crees importante que los estudiantes aprendan lo que has expuesto anteriormente (relevancia del tema o situación)?

3) ¿Conoces las posibles dificultades de aprendizaje de los niños o sus ideas alternativas sobre esta situación concreta?

4) ¿Conoces las dificultades o limitaciones en la enseñanza de los aspectos mencionados antes?

5) ¿Qué metodología de enseñanza utilizarías para obtener un mayor aprendizaje de los estudiantes en el caso planteado? ¿Qué actividades concretas plantearías? 
6) ¿Cómo evaluarías si los estudiantes han alcanzado realmente los objetivos planteados al principio?

\subsection{Condiciones del estudio}

El ReCo requiere determinar una situación o problema concreto al cual se refieren las preguntas del instrumento. Con el fin de explorar el efecto de la instrucción en didáctica de las ciencias en el Grado se consideraron varias situaciones en dos condiciones: una de Transferencia cercana (TC en adelante) y otra de Transferencia lejana (TL en adelante). La TC de conocimiento se produce entre un contexto de adquisición y otro de aplicación que son de muy similares características y que están estrechamente relacionados, mientras que la TL de conocimiento se da en aquellos casos en los que el contenido aprendido se aplica en contextos diferentes al de aprendizaje y con estímulos de distinta naturaleza (BARNARD, 2005).

Para el presente estudio se propusieron las siguientes situaciones:

1) Condición de transferencia cercana (TC). Las situaciones propuestas pertenecen a temas que se habían tratado en el Grado, tanto en la materia de formación en conocimiento científico, como en la materia sobre conocimiento en didáctica de las ciencias experimentales.

2) Condición de transferencia lejana (TL). Las situaciones propuestas se caracterizan por pertenecer a temas que, si bien se habían tratado en la materia de formación en conocimiento científico, no fueron trabajadas de forma explícita en la materia de didáctica de las ciencias experimentales.

Comparar los resultados de ambas condiciones permitirá valorar si la formación en didáctica de las ciencias es necesaria (en tanto los resultados en TL no se consideren adecuados), y suficiente (si los resultados en TC se consideran adecuados).

La Tabla 1 recoge ejemplos de situacionestemas propuestos en ambas condiciones:
Tabla 1. Ejemplos de situaciones propuestas en ambas condiciones del estudio

\section{Condición del estudio \\ Ejemplos de situaciones-temas propuestos}

$\begin{array}{ll}\text { TC: Transferencia } & \text {-Fases de la Luna. } \\ \text { cercana. } & \text {-Flotación de cuerpos en } \\ & \text { agua u otros líquidos. } \\ \text { TL: Transferencia } & \text {-La Luna orbitando } \\ \text { lejana. } & \text { alrededor de la Tierra. } \\ & \text {-Un algodón empapado en } \\ & \text { alcohol, ardiendo con } \\ & \text { llama. }\end{array}$

\subsection{Procedimiento de recogida de datos}

Primero se obtuvo permiso de los profesores/as de cada grupo intacto. Los datos se tomaron inmediatamente después de acabar la formación en didáctica de las ciencias obligatoria en el Grado. Los estudiantes fueron informados unos días antes y se solicitó su libre participación, que se situó entre un $90 \%$ y un $95 \%$ de los integrantes asignados oficialmente en cada grupo.

Los estudiantes fueron asignados al azar a una de las condiciones contempladas en este estudio exploratorio, TC (52 participantes) y TL (60 participantes). El investigador presente repartió un listado de temas a elegir (diferentes en las dos condiciones del estudio), y el instrumento en papel. Luego leyó en voz alta las instrucciones sobre la tarea a realizar y subrayó la necesidad de seleccionar libremente una de las temáticas ofrecidas (en cada condición). El tiempo para ejecutar la tarea se situó entre 45 y 55 minutos.

\section{RESULTADOS}

\subsection{Descripción y análisis de los datos}

\subsubsection{Determinación de indicadores}

En primer lugar, se pretendió reflejar una articulación del conocimiento didáctico en ciencias en aspectos fácilmente identificables por un experto (por ejemplo, formulación de objetivos con base conceptual, procedimental, y actitudinal, una relación coherente entre objetivos planteados, metodología y actividades a realizar, 
y evaluación, atención a la diversidad, etc.) Los indicadores expertos iniciales, fueron completados, matizados y re-elaborados recursivamente a partir de la variabilidad en las respuestas de los participantes.

En el Apéndice 1 se muestra el conjunto resultante de indicadores para evaluar el CD dentro del instrumento ReCo. Se incluyen respuestas-ejemplo asociadas con cada indicador.

\subsubsection{Satisfacción de los indicadores de calidad de $C D$}

El Apéndice 1 ofrece los porcentajes de participantes cuyas respuestas se asociaron con cada uno de los indicadores de CD. No se reflejó la cantidad de ideas expuestas, sino si una idea al menos satisface cada indicador. Porcentajes nulos en los indicadores "Otras ideas" en cada aspecto, indican la suficiencia del conjunto para dar cuenta de las respuestas de los/las participantes en este estudio exploratorio ( $\sin$ perjuicio de que los indicadores puedan y deban ser mejorados o redefinidos).

Entre los resultados podemos destacar:

Objetivos formulados.- Casi la totalidad de los sujetos participantes $(96,4 \%)$ nombra objetivos conceptuales, pero pocos nombran objetivos procedimentales y actitudinales $(20,5 \%$ y $2,7 \%$ respectivamente). La naturaleza de las ciencias o la evolución histórica de las ideas apenas se consideran. Globalmente, un $13,4 \%$ de futuros maestros explicitó algún error conceptual.

Relevancia o importancia educativa.- La utilidad en la vida diaria es el indicador con mayor presencia $(69,6 \%)$, y luego la relación del tema o situación con otros contenidos (24,1\%).

Dificultades de aprendizaje.- Las ideas previas erróneas de los niños están muy presentes $(81,3 \%)$, seguidas de la dificultad para construir conceptos abstractos (34,8\%) o superar un estado de actitud negativa o falta de motivación hacia la ciencia $(18,8 \%)$. Las dificultades debidas a destrezas intelectuales poco desarrolladas están poco presentes $(7,1 \%)$.

Dificultades de enseñanza.- Los obstáculos más nombrados son hacer entender conceptos abstractos $(49,1 \%)$, superar ideas erróneas o el bajo conocimiento previo $(47,3 \%)$, y falta de recursos necesarios (33,9\%). También se tienen en cuenta la desmotivación o actitudes negativas de los niños/as (22,3\%) y la falta de tiempo $(15,2 \%)$. Las dificultades asociadas a falta de preparación o de competencias apropiadas del propio maestro (10,7\%) son poco nombradas .

Metodología y Actividades.- Una amplia mayoría de participantes $(86,6 \%)$ menciona algún enfoque general del modo de instrucción, como fomentar el aprendizaje significativo 0 la utilización de una metodología activa o basada en actividad experimental. Sin embargo, sólo el $26,8 \%$ aporta argumentos para sostener sus propuestas. Sólo el $17,0 \%$ de participantes explicita partir del conocimiento previo de los niños/as. Algo más de la mitad de todos los participantes $(51,8 \%)$ propone actividades concretas relacionadas con el tema específico planteado. Una pequeña minoría (5,4\%) menciona las necesidades de organización y gestión de los estudiantes en el aula (como trabajo cooperativo).

Evaluación.- Nada más el 13,4\% de participantes relaciona la evaluación con los objetivos propuestos. Se mencionan indicadores para evaluar $(33,9 \%)$ basados en la participación, el comportamiento, el interés o el progreso durante el curso. Frecuentemente estos indicadores no fueron mencionados en los objetivos planteados. Un gran número de encuestados $(67,9 \%)$ nombró técnicas y materiales para evaluar (exámenes, trabajos prácticos, exposiciones públicas, experimentos, etc.), pero sólo una parte menor $(22,3 \%)$ explicitó actividades para evaluar lo pretendido, o fue capaz de dar argumentos para sustentar sus propuestas (23,2\%).

En suma, una gran mayoría de participantes fue capaz de aportar ideas relevantes en cada aspecto del instrumento (entre un $0 \%$ y un $3,6 \%$ expresó únicamente ideas vagas, sin aportación real).

\subsubsection{Efectos de la instrucción específica en didáctica de las ciencias}

La comparación de los resultados obtenidos en las dos condiciones del estudio propuestas (Transfer cercano, TC y Transfer lejano, TL) muestra diferencias en distintos indicadores. Las más notables son:

Formulación de objetivos.- Resultaron evidentes las diferencias en objetivos procedimentales propuestos (TC: $36,5 \%$; TL: 6,7\%) y en objetivos actitudinales (TC: 5,8\%; TL: $0,0 \%)$ y 
epistemológicos (TC: 1,9\% TL: 0,0\%) aunque con porcentajes bajos en ambas condiciones. Importante es que los errores conceptuales se dieran únicamente en la condición TL $(25,0 \%$; TC: $0 \%)$.

Relevancia o importancia educativa.- Más sujetos en la condición TC fueron capaces de establecer relaciones entre los temas tratados con otros contenidos curriculares (TC: $34,6 \%$; TL: $15,0 \%)$.

Dificultades de aprendizaje.- La dificultad de superar una actitud o motivación negativa preocupó más en la condición TL (TC: 1,9\%; TL: $33,3 \%)$.

Dificultades de enseñanza.- Un mayor porcentaje de participantes en la condición TL manifestaron dificultades relacionadas con cómo corregir las ideas erróneas de los niños (TC: 30,8\%; TL: $61,7 \%$ ), con la superación de una baja motivación (TC: 7,7\%; TL: 35,0\%) o con características negativas del contexto (TC: $5,8 \%$; TL: 45,0\%). Un mayor porcentaje de estudiantes en la condición TC señaló la falta de tiempo como obstáculo instruccional (TC: $23,1 \%$; TL: $8,3 \%)$, tal vez conscientes de que ciertas tareas de aprendizaje requieren tiempo para su desarrollo (las experimentales).

Metodología y Actividades.- Las principales diferencias se manifestaron al comparar la presencia o ausencia de actividades específicas del tema en cuestión: (TC: $96,2 \%$; TL: 13,3\%). Los porcentajes se desequilibraron al contrario (TC: $1,9 \%$; TL: $23,3 \%$ ) al proponer tipos de tareas, pero sin concretarlas (observar, experimentar, discutir, brainstorming, etc.), y al sugerir genéricamente partir del conocimiento previo de los estudiantes (TC: $5,8 \%$ TL: $26,7 \%$ ), pero sin especificar ninguna actividad sobre cómo hacerlo.

Evaluación.- No se apreciaron diferencias entre condiciones dignas de mención.

\section{DISCUSIÓN Y CONCLUSIONES}

Respecto al primer objetivo, el conjunto resultante de "rúbricas" ha mostrado suficiencia para dar cuenta de las respuestas de nuestros participantes. No obstante, debemos recordar que el conjunto de indicadores no pretende proponer un modelo teórico particular sino ser útil y, por tanto, está sujeto a revisión y mejora por parte de toda la comunidad de investigadores/as en el área.
El segundo objetivo, usar los indicadores para evaluar el conocimiento básico en didáctica de las ciencias de futuros/as maestros/as, produjo algunos resultados estimulantes, como los bajísimos porcentajes de ideas vagas sin aportación real entre los participantes (ver Apéndice 1). Sin embargo, en muchas ocasiones las ideas aportadas muestran bajos niveles de profundidad y falta de concreción y adecuación al tema (poca especificidad).

Se revelaron carencias importantes en porcentajes significativos de participantes. Entre ellas:

a) Superficialidad de las propuestas, que se manifiesta en la declaración de "buenas intenciones", pocas veces acompañadas de profundidad didáctica. Con frecuencia, los alumnos utilizan "palabras clave" que luego no se materializan en propuestas concretas coherentes con lo declarado. Afirmaciones como "Usaría una metodología en la que el alumno tenga el papel activo del proceso de aprendizaje y el maestro sea únicamente su guía...para que el alumno llegue al conocimiento de forma autónoma... el aprendizaje sea más significativo y perdure" (participante \#26A), se acompañaron pocas veces de actividades que las concretaran y ejemplificaran. Cuando ello sí sucedió, a menudo se trató de actividades más bien tradicionales propuestas bajo la suposición de que una simple ejecución autónoma por los niños significa, automáticamente, un "papel activo" del alumno o un "aprendizaje significativo". Por ejemplo: "observación de la luna durante el ciclo de 28 días y dibujar en un cuaderno en qué fase se encuentra cada día..."; "creación de una maqueta del sistema solar..." (el mismo participante \#26A). También Ozden et al. (2013) encontraron que la mayoría del profesorado de primaria carece de conocimientos didácticos apropiados sobre las actividades y sobre los recursos más pertinentes para la enseñanza de un tema científico. Esto sugiere que la conexión entre conocimiento disciplinar y conocimiento didáctico (dentro del CDC pretendido) debe ser mejorada para que los futuros maestros conozcan el valor educativo de cada tema en particular y sean precisos a la hora de plantear actividades que los pongan de relieve. Ello requiere de un cierto nivel de dominio disciplinar en variados temas de ciencias que, probablemente, no es el alcanzado por la mayoría de este alumnado (pese a la asignatura cursada). 
b) Poca comprensión del currículo de ciencias y de sus posibilidades educativas, puesta de manifiesto al tratar de sustentar con argumentos la relevancia educativa del tema o situación. Claro está que los (futuros) maestros han de asumir currículos que les son impuestos y tienen poco margen de discusión sobre ellos. Si esto se une a que su formación científica y didáctica queda muchas veces en niveles más bajos de lo deseable, el resultado podría esperarse. La relación entre un bajo conocimiento de ciencias y dificultades para concebir una buena enseñanza de las ciencias ha sido encontrada repetidamente (véase, por ejemplo, DAVIS y PETISH, 2005).

c) Escasa consideración de objetivos procedimentales, actitudinales o epistemológicos. Muchos participantes olvidaron que la construcción activa del conocimiento en ciencias implica algo más que dominio de los conceptos, lo cual los acerca a posturas tradicionales, en contra del aparente constructivismo que muchas veces ellos mismos declaran (HAMED et al., 2016). Los formadores de maestros deberían considerar la posibilidad de que ello se deba a las vivencias de estos participantes en las aulas de ciencias durante su vida estudiantil, incluyendo el Grado universitario. Este problema no sólo aparece en los futuros maestros de primaria: Ravanal y López-Cortés (2016) también encontraron que los futuros profesores de biología focalizaban el CDC en el conocimiento conceptual sobre todo.

d) Desvinculación entre objetivos, actividades de enseñanza, y evaluación. Musikul (2007) observó también limitaciones en las estrategias de enseñanza de las ciencias y sobre evaluación en profesores con un conocimiento poco profundo de la ciencia. La falta de conocimiento profundo de la ciencia podría estar dando impulso a una concepción "activista" de la profesión con baja coherencia, basada en "piezas a ejecutar" sin conexión argumentada, sin metas instruccionales claras y, por tanto, sin vías definidas para alcanzarlas.

En cuanto al tercer objetivo, se apreciaron diferencias entre los estudiantes a quienes se ofrecieron situaciones de ciencias cercanas a las previamente trabajadas en didáctica de las ciencias (condición de transferencia cercana, TC), y aquellos cuyas situaciones propuestas no tratadas antes en esta materia (condición de transferencia lejana, TL). Los participantes en la condición TC, aportaron más ideas focalizadas y adaptadas a la situación elegida que los de la condición TL, y concretaron actividades para el aprendizaje de la situación planteada con objetivos más variados y apropiados (mayor adecuación y especificidad).

Otro efecto positivo no esperado es que una cuarta parte de los estudiantes en la condición TL manifestaron errores conceptuales, pero éstos no aparecieron en la condición TC. Probablemente, el cambio de meta u objetivo desde "aprender para saber" a "aprender para enseñar" supone un esfuerzo metacognitivo que hace conscientes a los futuros maestros en condición TC de sus errores y obstáculos de comprensión, y les obliga a darles solución.

En resumen, en comparación con la formación en didáctica general, la formación en didáctica de las ciencias parece producir un aprendizaje específico que es necesario en los futuros maestros y maestras. Sin embargo, esa misma comparación hace ver que la formación específica impartida no es suficiente como para garantizar que los futuros maestros sabrán cómo afrontar situaciones nuevas de enseñanzaaprendizaje de las ciencias. La transferencia del conocimiento didáctico a temas no trabajados explícitamente es pobre en aspectos de gran importancia, como la formulación de objetivos de tipo variado (procedimentales, epistémicos), y el diseño de actividades que incidan directa y concretamente sobre los objetivos planteados. Si esta transferencia "lejana" no mejora, los maestros no estarán preparados para afrontar los muchos años de servicio que tienen por delante, en donde los cambios en el currículo de ciencias y tecnología son inevitables y necesarios.

La solución puede centrarse en eliminar obstáculos en ese proceso de transferencia y generalización durante el Grado universitario:

a) Mejorar el dominio del contenido de ciencias básicas, a través de claves como la elaboración, validación y estructuración conceptual de la ciencia, las relaciones causaefecto de las leyes, la comprensión de fenómenos sin la mediación de la matemática, y sobre todo, con la realización de experimentos sencillos.

b) Proporcionar mejores recursos, tanto para el análisis didáctico (uso inteligente de mapas o redes conceptuales, conocimiento y detección de obstáculos de comprensión posibles, inferencias necesarias, activación de conocimiento previo, elaboración de 
representaciones mentales demasiado abstractas, etc.), como para la acción didáctica, convirtiendo terminología vacía en "saber hacer" profesional (actividades centradas en objetivos concretos y variados, simples, adaptados al tema y evaluables). El análisis didáctico y la acción didáctica se han mostrado como los dos factores constitutivos del conocimiento didáctico en ciencias (VERDUGO-PERONA et al., 2017).

c) Incidir en aspectos meta-didácticos y profesionalizadores durante la formación en el Grado universitario, como es el caso de un lenguaje del oficio unificado, en donde se habla de formulación de objetivos o de coherencia entre objetivos, actividades y evaluación (para exigirla, naturalmente), y, sobre todo, poner más a los futuros profesores en situación de producción (impartir clase) y menos en situación de asimilación pasiva (recibir clase).

Al parecer, el conocimiento didáctico requiere comprensión de los fundamentos instruccionales que, hoy por hoy, se ofrecen como una suma compleja de conocimiento científico y artesanal que, unido a la variedad de disciplinas que los futuros maestros han de dominar, hace extraordinariamente difícil adquirir una formación coherente y sólida. Quizás la formación de maestros deba realizar esfuerzos mayores en la concreción de actividades formativas que den forma, profundidad coherencia y realidad a las distintas orientaciones instruccionales "declaradas" en las aulas.

Debemos insistir en que todos los resultados obtenidos suponen simplemente una exploración con una muestra no representativa de estudiantes de magisterio en una universidad pública particular, y con un diseño de investigación que puede ser optimizado. Por tanto, estos resultados no son aún generalizables y requieren validación adicional.

\section{REFERENCIAS}

1. Abd-El-Khalick, F. Eurasia Journal of Mathematics, Science and Technology Education, 2006, 2, 1.

2. Alonzo, A. C., Kobarg M., Seidel T. Journal of Research in Science Teaching, 2012, 49, 1211.

3. Barnard, J. K. Tesis Doctoral, The Ohio State University, USA, 2005.

4. Barnett, J., Hodson, D. Science
Education, 2001, 85, 426.

5. Brines, M., Solaz-Portolés J.J, Sanjosé, V. Enseñanza de las Ciencias, 2016, 34, 107.

6. Davis, E. A., Petish, D. Journal of Science Teacher Education, 2005, 16, 263.

7. Eames, C., Williams, J., Hume, A., Lockley, J., Wellington, J. Teaching and Learning Research Initiative (TLRI), 2011. http://www.tlri.org.nz/sites/default/files/proj ects/9289_summaryreport.pdf, consultado en marzo 2017.

8. Faikhamta, C., Clarke, A. Research in Science Education, 2013, 43, 955.

9. Gardner, A.L., Gess-Newsome, J. Paper presented at the Annual Meeting of the National Association for Research in Science Teaching. Orlando, FL, 2011, http://www.bscs.org/sites/default/files/leg acy/pdf/Community_Sessions_NARST20 11_PCK\%20Rubric\%20Paper.pdf, consultado en marzo 2017.

10. Hamed, S., Rivero, A., Martín del Pozo, R. Revista Eureka sobre Enseñanza $y$ Divulgación de las Ciencias, 2016, 13, 476. http://hdl.handle.net/10498/18301, consultado en abril 2017.

11. Henze, I., van Driel J. H., Verloop, N. International Journal of Science Education, 2008, 30, 1321.

12. Hume, A.; Berry, A. Research in Science Education, 2011, 41, 341.

13. Jüttner, M., Boone W., Park S., Neuhaus B. J. Educational Assessment Evaluation and Accountability, 2013, 25, 45.

14. Jüttner, M., Neuhaus, B. J. Journal of Education and Training Studies, 2013, 1, 113.http://redfame.com/journal/index.php/j ets/article/view/126/132, consultado en abril 2017.

15. Käpylä M., Heikkinen J. P., Asunta T. International Journal of Science Education, 2009, 31, 1395.

16. Kleickmann, T., Richter, D., Kunter, M., Elsner, J., Besser, M., Krauss, S., Baumert, J. Journal of Teacher Education, 2013, 64, 90.

17. Krauss, S., Brunner, M., Kunter, M., Baumert, J., Blum, W., Neubrand, J., Jordan, A. Journal of Educational Psychology, 2008, 100, 716.

18. Loughran, J., Berry, A., Mulhall, P. Understanding and Developing Science Teachers' Pedagogical Content Knowledge. Rotterdam, the Netherlands: Sense Publishers, 2006. 
19. Loughran, J., Mulhall, P., Berry, A. International Journal of Science Education, 2008, 30, 1301.

20. Malcolm S.A., Mavhunga E. 23rd Annual Conference of the Southern African Association for Research in Mathematics, Science and Technology Education. Maputo, Mozambique. 2015, https://www.academia.edu/10099974/The _Development_and_Validation_of_an_Ins trument_Measuring_Topic_Specific_PCK _in_Stoichiometry_Preliminary_Findings, consultado en abril 2017.

21. Manizade, A.G., Mason M.M. Educational Studies in Mathematics, 2011, 76, 183.

22. Magnusson, S., Krajcik, J., Borko, H. En Examining Pedagogical Content Knowledge; Gess-Newsome, J., Lederman, N.G. (Eds.) Examining Pedagogical Content Knowledge. Dordrecht, the Netherlands: Kluwer Academic, 1999, pp. 95-132.

23. Morrison, A. D., Luttenegger, K. C. The Qualitative Report, 2015, 20, 804. http://www.nova.edu/ssss/QR/QR20/6/mo rrison1.pdf, consultado en abril 2017.

24. Musikul, K.. Tesis Doctoral, University of Missouri-Columbia, USA, 2007. https://mospace.umsystem.edu/xmlui/han dle/10355/4667, consultado en enero 2017

25. Nilsson, P. International Journal of Science Education, 2008, 30, 1281.

26. Ozden, M., Usak, M., Ulker, R., Sorgo, A. Journal of Environmental Protection and Ecology, 2013, 14, 1432.

27. Park, S., Chen Y-C. Journal of Research in Science Teaching, 2012, 49, 922.

28. Park, S., Chen, Y-C., Jang, J. International conference of the Association for Science Teacher Education, St. Louis, MI. 2008.

29. Ravanal, E., López-Cortés, F. Revista Eureka sobre Enseñanza y Divulgación de las Ciencias, 2016, 13, 725. http://hdl.handle.net/10498/18508, consultado en abril 2017.

30. Shulman, L. S. Harvard Educational Review, 1987, 57, 1.

31. Verdugo-Perona, J.J., Olmos, R., SolazPortolés, J.J., Sanjosé, V. Interciencia, 2017, 42, 446.

32. Zeidler, D.L. Journal of Science Teacher Education, 2002, 13, 27. 
Apéndice 1. Indicadores específicos para evaluar el Conocimiento Didáctico en ciencias, porcentaje de participantes que los satisfacen y ejemplos de respuestas dadas en cada indicador.

\begin{tabular}{|c|c|c|c|}
\hline & Indicadores & $\%$ & Ejemplos de respuesta \\
\hline \multirow{7}{*}{$\begin{array}{l}\text { Formulación } \\
\text { de objetivos }\end{array}$} & $\begin{array}{l}\text { Se explicitan objetivos } \\
\text { conceptuales en relación con la } \\
\text { situación seleccionada }\end{array}$ & 96,4 & $\begin{array}{c}\text { "(Aprender) la necesidad de oxígeno en } \\
\text { cualquier combustión” \#3A }\end{array}$ \\
\hline & $\begin{array}{l}\text { Se explicitan objetivos } \\
\text { procedimentales en relación con } \\
\text { la situación seleccionada }\end{array}$ & 20,5 & $\begin{array}{c}\text { "Observar que un globo no se puede } \\
\text { hinchar dentro de una botella llena de aire" } \\
\# 18 B\end{array}$ \\
\hline & $\begin{array}{l}\text { Se explicitan objetivos } \\
\text { actitudinales o valores. }\end{array}$ & 2,7 & $\begin{array}{c}\text { "Adoptar una postura crítica frente a las } \\
\text { situaciones que se nos puedan presentar en } \\
\text { relación con los materiales conductores y no } \\
\text { conductores" \#61B }\end{array}$ \\
\hline & $\begin{array}{l}\text { Se menciona la naturaleza de la } \\
\text { ciencia o historia de la ciencia }\end{array}$ & 0,9 & $\begin{array}{l}\text { "Aprender que la ciencia está presente en } \\
\text { todos los ámbitos del día a día" \#26B }\end{array}$ \\
\hline & Generales e imprecisos & 1,8 & "Experimentar con diferentes objetos" \#8B \\
\hline & $\begin{array}{l}\text { Los sujetos muestran errores } \\
\text { conceptuales }\end{array}$ & 13,4 & $\begin{array}{c}\text { "Que durante la combustión el alcohol etílico } \\
\text { se ha evaporado" \#10A }\end{array}$ \\
\hline & Ideas no contempladas* & 0 & \\
\hline \multirow{4}{*}{$\begin{array}{l}\text { Importancia } \\
\text { educativa }\end{array}$} & $\begin{array}{l}\text { Relación con utilidad en la vida } \\
\text { diaria. }\end{array}$ & 69,6 & $\begin{array}{c}\text { "Creo que es algo que les resultaría útil en } \\
\text { el ámbito cotidiano" \#85A }\end{array}$ \\
\hline & $\begin{array}{l}\text { Importancia en la formación } \\
\text { personal. }\end{array}$ & 33,0 & $\begin{array}{l}\text { "Considero que es un mínimo para ser } \\
\text { alfabeto científicamente" \#172A }\end{array}$ \\
\hline & $\begin{array}{l}\text { Relación, prelación y secuencia } \\
\text { entre estos y otros contenidos. }\end{array}$ & 24,1 & $\begin{array}{l}\text { "Estos conceptos pueden ayudar a los niños } \\
\text { a entender otros conceptos en un futuro, } \\
\text { que probablemente sin entender por qué } \\
\text { unos objetos flotan y otros se hunden no } \\
\text { podrían entender" \#1B }\end{array}$ \\
\hline & Ideas no contempladas* & 0 & \\
\hline \multirow{5}{*}{$\begin{array}{l}\text { Dificultades } \\
\text { de } \\
\text { aprendizaje }\end{array}$} & $\begin{array}{l}\text { Conocimiento previo insuficiente } \\
\text { o erróneo. }\end{array}$ & 10,7 & $\begin{array}{c}\text { "Entender el concepto en sí (...) implica } \\
\text { tener gran cantidad de conocimientos } \\
\text { previos que deben de estar bien asentados. } \\
\text { Y eso en muchas ocasiones no ocurre así." } \\
\text { \#49A }\end{array}$ \\
\hline & $\begin{array}{l}\text { Incapacidad para explicitar, } \\
\text { abandonar ideas erróneas o } \\
\text { superar percepciones } \\
\text { sensoriales. }\end{array}$ & 81,3 & $\begin{array}{c}\text { "Las ideas previas suelen ser para ellos (los } \\
\text { estudiantes) algo correcto y es difícil aceptar } \\
\text { nuevos conocimientos dejando a un lado los } \\
\text { adoptados anteriormente" \#141A }\end{array}$ \\
\hline & $\begin{array}{l}\text { Nivel de abstracción de } \\
\text { determinados conceptos. }\end{array}$ & 34,8 & $\begin{array}{l}\text { "La principal dificultad para ellos es que no } \\
\text { pueden ver lo que se les enseña" \#14B }\end{array}$ \\
\hline & $\begin{array}{l}\text { Incapacidad para desarrollar } \\
\text { habilidades procedimentales. }\end{array}$ & 0 & \\
\hline & $\begin{array}{l}\text { Superar actitud/motivación } \\
\text { negativa hacia la ciencia. }\end{array}$ & 18,8 & $\begin{array}{l}\text { "Un exceso de motivación, igual que una } \\
\text { falta, puede ser una dificultad ya que genera } \\
\text { unas expectativas muy altas y una gran }\end{array}$ \\
\hline
\end{tabular}




\begin{tabular}{|c|c|c|c|}
\hline & \multicolumn{3}{|r|}{$\begin{array}{c}\text { desilusión o apatía ante los resultados" } \\
\# 96 \mathrm{~A}\end{array}$} \\
\hline & $\begin{array}{l}\text { Dificultades para comprender la } \\
\text { progresión histórica. }\end{array}$ & 0 & \\
\hline & $\begin{array}{l}\text { Diversidad en el alumnado / Bajo } \\
\text { desarrollo de capacidades } \\
\text { cognitivas }\end{array}$ & 7,1 & $\begin{array}{l}\text { "Debe haber una atención a la diversidad } \\
\text { para poner dicho aprendizaje a la altura de } \\
\text { todos los alumnos individualmente" \#141A }\end{array}$ \\
\hline & $\begin{array}{l}\text { Aceptar/entender explicación de } \\
\text { la ciencia actual. }\end{array}$ & 0 & \\
\hline & Ideas no contempladas* & 0 & \\
\hline \multirow{11}{*}{$\begin{array}{l}\text { Dificultades } \\
\text { de } \\
\text { enseñanza }\end{array}$} & $\begin{array}{l}\text { Hacer entender conceptos } \\
\text { abstractos o dificultosos. }\end{array}$ & 49,1 & $\begin{array}{l}\text { Las dificultades de enseñanza están } \\
\text { bastante relacionadas con las de } \\
\text { aprendizaje, ya que vuelven a pasar por la } \\
\text { necesidad de explicar los fenómenos } \\
\text { científicos de una forma abstracta" \#15B }\end{array}$ \\
\hline & $\begin{array}{l}\text { Superar bajo conocimiento previo } \\
\text { y/o misconceptions. }\end{array}$ & 47,3 & $\begin{array}{l}\text { "Las dificultades que podría destacar (...) } \\
\text { que el profesor no conozca las ideas previas } \\
\text { de los alumnos sobre el tema y no tenga } \\
\text { claro por dónde empezar para no confundir } \\
\text { a los alumnos" \#32A }\end{array}$ \\
\hline & $\begin{array}{l}\text { Realizar experimentos o } \\
\text { actividades procedimentales } \\
\text { apropiadas. }\end{array}$ & 0,9 & $\begin{array}{l}\text { "La dificultad que puede encontrar un } \\
\text { maestro (...)...si los maestros realizaran } \\
\text { actividades prácticas donde los alumnos } \\
\text { participen....aprenderán de una manera casi } \\
\text { definitiva" \#22B }\end{array}$ \\
\hline & $\begin{array}{l}\text { Captar la atención y mantenerla; } \\
\text { despertar interés, actitud del } \\
\text { maestro. }\end{array}$ & 22,3 & $\begin{array}{l}\text { "(Otro problema es) que el experimento no } \\
\text { sea atractivo para ellos y no capte su } \\
\text { atención..." \#27A }\end{array}$ \\
\hline & Usar bien la historia de la ciencia. & 0 & \\
\hline & $\begin{array}{l}\text { Comunicación y uso de } \\
\text { vocabulario difícil. }\end{array}$ & 0 & \\
\hline & $\begin{array}{l}\text { Falta de recursos, lugares y } \\
\text { materiales de laboratorio. }\end{array}$ & 33,9 & $\begin{array}{l}\text { "Las limitaciones más frecuentes vienen } \\
\text { dadas por los recursos... bastante } \\
\text { escasos... viéndonos obligados a } \\
\text { comprarlos por nuestra parte" \#14B }\end{array}$ \\
\hline & $\begin{array}{l}\text { Falta de tiempo para desarrollar } \\
\text { los contenidos. }\end{array}$ & 15,2 & $\begin{array}{l}\text { "También puede influir (en la dificultad de } \\
\text { enseñar) el tiempo que se pueda dedicar (al } \\
\text { tema)" \#26A }\end{array}$ \\
\hline & $\begin{array}{l}\text { Falta de preparación o de } \\
\text { cualidades del propio maestro. }\end{array}$ & 10,7 & $\begin{array}{l}\text { "En mi opinión los alumnos no deben pagar } \\
\text { su mal carácter o su mala organización" } \\
\# 28 \text { A }\end{array}$ \\
\hline & $\begin{array}{l}\text { Dificultades contextuales: } \\
\text { inmobiliario, peligrosidad con } \\
\text { niños, etc... }\end{array}$ & 26,8 & $\begin{array}{l}\text { "No siempre los colegios disponen de } \\
\text { laboratorios o aulas habilitadas y materiales } \\
\text { para realizar este tipo de demostraciones de } \\
\text { forma segura..." \#53A }\end{array}$ \\
\hline & $\begin{array}{l}\text { Sólo ideas vagas, sin aportación } \\
\text { real }\end{array}$ & 0 & \\
\hline
\end{tabular}




\begin{tabular}{|c|c|c|c|}
\hline & Ideas no contempladas* & 0 & \\
\hline \multirow{9}{*}{$\begin{array}{l}\text { Metodología } \\
\text { de } \\
\text { enseñanza }\end{array}$} & $\begin{array}{l}\text { Este tema no lo enseñaría } \\
\text { porque es inadecuado. }\end{array}$ & 0 & \\
\hline & $\begin{array}{l}\text { Enfoque general de instrucción } \\
\text { (activa, constructivista, etc.). }\end{array}$ & 86,6 & $\begin{array}{l}\text { "Una metodología crítica y reflexiva que } \\
\text { permita a los niños cuestionarse su } \\
\text { conocimiento..." \#16B }\end{array}$ \\
\hline & $\begin{array}{l}\text { Argumentos a favor/ en contra de } \\
\text { ciertas metodologías. }\end{array}$ & 26,8 & $\begin{array}{l}\text { "...Realización de actividades en primera } \\
\text { persona. Estos temas tan densos y difíciles, } \\
\text { si los enseñamos solo dando teoría y los } \\
\text { alumnos no descubren el porqué de la } \\
\text { flotación, son temas difíciles de entender" } \\
\text { \#95B }\end{array}$ \\
\hline & $\begin{array}{l}\text { Organización: roles de maestro y } \\
\text { de estudiantes, ambiente, etc. }\end{array}$ & 5,4 & $\begin{array}{l}\text { "Trabajar en grupo podría motivarles (en } \\
\text { este tema (Tierra-Luna)" \#8A }\end{array}$ \\
\hline & $\begin{array}{l}\text { Partir del conocimiento previo del } \\
\text { alumno }\end{array}$ & 17,0 & $\begin{array}{l}\text { "(Que los alumnos) construyan su } \\
\text { conocimiento desde sus conocimientos } \\
\text { previos..." \#72A }\end{array}$ \\
\hline & $\begin{array}{l}\text { Tipología de tareas (observar, } \\
\text { experimentar, discutir, } \\
\text { brainstorming, etc...). }\end{array}$ & 13,4 & $\begin{array}{l}\text { "Aplicar el método científico: planteamiento } \\
\text { de hipótesis-experimentación - verificación" } \\
\# 79 A\end{array}$ \\
\hline & $\begin{array}{l}\text { Actividades concretas asociadas } \\
\text { con objetivos planteados para } \\
\text { este tema }\end{array}$ & 51,8 & $\begin{array}{l}\text { "Utilizaría dos bolas de corcho (Tierra y } \\
\text { Luna) y un foco encendido (Sol)... } \\
\text { Explicaría el movimiento lunar y pediría a } \\
\text { los alumnos que lo pusieran en práctica" } \\
\text { \#15B }\end{array}$ \\
\hline & $\begin{array}{l}\text { Sólo ideas vagas sin aportación } \\
\text { real. }\end{array}$ & 3,6 & $\begin{array}{l}\text { "Favorecer al alumnado de material tangible } \\
\text { para ellos que les haga comprender en su } \\
\text { totalidad el tema abarcado (sic)". \#112A }\end{array}$ \\
\hline & Ideas no contempladas* & 0 & \\
\hline \multirow{5}{*}{ Evaluación } & $\begin{array}{l}\text { La evaluación se relaciona con } \\
\text { los objetivos planteados al inicio } \\
\text { y/o con la metodología. }\end{array}$ & 13,4 & $\begin{array}{l}\text { "No tendría sentido que, habiendo seguido } \\
\text { una metodología de enseñanza práctica o } \\
\text { experimental, evaluara a mis alumnos con } \\
\text { un examen oral o escrito" \#90B }\end{array}$ \\
\hline & $\begin{array}{l}\text { Orientación general de la } \\
\text { evaluación. }\end{array}$ & 25,9 & $\begin{array}{l}\text { “(Realizaría) una evaluación continua donde } \\
\text { contaría el trabajo y el interés por mejorar } \\
\text { del alumno". \#61A }\end{array}$ \\
\hline & $\begin{array}{l}\text { Formas de evaluar, técnicas e } \\
\text { instrumentos. }\end{array}$ & 67,9 & $\begin{array}{l}\text { "Pasar un test antes de explicar }(\ldots) \text { y } \\
\text { repetirlo al final para saber lo que han } \\
\text { aprendido" \#156A }\end{array}$ \\
\hline & $\begin{array}{l}\text { Justificación y argumentos sobre } \\
\text { evaluación. }\end{array}$ & 23,2 & $\begin{array}{l}\text { "(Haría preguntas mientras ellos realizan } \\
\text { experimentos). Lo haría así porque de esta } \\
\text { forma los niños estarían centrados en la } \\
\text { tarea y no despistados... y no tienen } \\
\text { presión..." \#97B }\end{array}$ \\
\hline & $\begin{array}{l}\text { Indicadores o aspectos concretos } \\
\text { a evaluar: las ideas previas, etc. }\end{array}$ & 33,9 & $\begin{array}{l}\text { "Llevaría un control diario del interés, } \\
\text { capacidad de abstracción y generalización y } \\
\text { participación en los debates (de cada niño)". } \\
\text { \#16B }\end{array}$ \\
\hline
\end{tabular}


Actividades de evaluación concretas.

Rechazan realizar examen.

Sólo ideas vagas sin aportación real.
22,3 "Les daría unos papeles que representaran las cargas positivas y negativas, y (pediría) que supieran describir su movimiento" \#88B

10,7 "Creo que toda evaluación que no conste de un examen escrito es constructiva..." \#26B

0,9 "Mediante una actividad donde demuestren lo aprendido. Igualmente necesitaría conocer al grupo (..) para poder realizar una evaluación que respondiera a sus necesidades" \#1A

${ }^{*}$ ) En el conjunto de aportaciones de los participantes no aparecieron ideas que no estuvieran contenidas ya en los indicadores de cada apartado.

PERIÓDICO TCHÊ QUÍMICA • www.periodico.tchequimica.com• Vol. 15 N. 29. 